\title{
Mood Extraction Using Facial Features to Improve Learning Curves of Students in E-Learning Systems
}

\author{
Abdulkareem Al-Alwani \\ Computer Science \& Engineering Department \\ Yanbu University College, Royal Commission Institute \& Colleges \\ Yanbu, Saudi Arabia
}

\begin{abstract}
Students' interest and involvement during class lectures is imperative for grasping concepts and significantly improves academic performance of the students. Direct supervision of lectures by instructors is the main reason behind student attentiveness in class. Still, there is sufficient percentage of students who even under direct supervision tend to lose concentration. Considering the e-learning environment, this problem is aggravated due to absence of any human supervision. This calls for an approach to assess and identify lapses of attention by a student in an e-learning session. This study is carried out to improve student's involvement in e-learning platforms by using their facial feature to extract mood patterns. Analyzing themoods based on emotional states of a student during an online lecture can provide interesting results which can be readily used to improvethe efficacy of content delivery in an e-learning platform. A survey is carried out among instructors involved in e-learning to identify most probable facial features that represent the facial expressions or mood patterns of a student. A neural network approach is used to train the system using facial feature sets to predict specific facial expressions. Moreover, a data association based algorithm specifically for extracting information on emotional states by correlating multiple sets of facial features is also proposed. This framework showed promising results in inciting student's interest by varying the content being delivered.Different combinations of interrelated facial expressions for specific time frames were used to estimate mood patterns and subsequently level of involvement of a student in an e-learning environment.The results achieved during the course of research showed that mood patterns of a student provide a good correlation with his interest or involvement during online lectures and can be used to vary the content to improve students' involvement in the e-learning system.More facial expressions and mood categories can be included to diversify the application of the proposed method.
\end{abstract}

Keywords-Mood extraction; Facial features; Facial recognition; Online education; E-Learning; Attention state; Learning styles

\section{INTRODUCTION}

The main problem arises in E-Learning as there is no supervisor to assess how students are physically and emotionally responding to the delivered content. Usually when the students taking any course online, they may lose concentration and focus resulting in poor academic performance. Tackling this issue can advance the e-learning process many fold as each student's interest can be assessed and necessary improvements can be made to the content to engage the user during the online lecture. In order to circumvent the problem of observing student on an e-learning platform, this research is conducted with a view to analyze the relationship between facial expressions of a student enrolled in an e-learning system and the ways to improve upon learning attitude of such students using information extracted from these features.

E-learning is a medium for imparting education anytime and anywhere, and due to recent advances in information technology, online education systems can be considered as a blessing and an important information technology asset. Knowledge transfer via informational technology tools requires careful planning and execution as the learning environment provided to the student during e-learning offers complex insight on the student's learning curve. In order to improve the e-learning experience, the process of learning becomes imperative as it majorly governs how much and how well a student can absorb knowledge during online lectures [1].Delivery of content, examinations and student feedback are important measures that have a direct effect on the learning curve of students as well as the e-learning objectives. Still the time frame required for relating and observing all these measures must be long enough to account for every possible detail [2].

These measures are also the same as that are used in traditional or on campus learning where teacher has a direct interaction with students. Initially computers and information technology was used as tools to improvise learning. This concept subsequently evolved to full-fledged e-learning systems. Universities have now started offering online courses and have developed e-learning platforms catering to the need of almost any student. E-learning has allowed off campus students to get educated at homes or simply anywhere in the world.

Knowledge delivery through e-learning offers numerous advantages but most of its features can only be fully utilized if the student's involvement and interest remains continuous throughout the course of online education [3].As a student has a personal preference for acquiring knowledge at one's own time and pace, this allows people from all walks of life to have an opportunity to learn and educate themselves without any restrictions of time and space.

With this evolution in the e-learning technologies and the increasing number of students, requirements for improving online education experience are getting more and more demanding. It's understood that more in depth studies are 
needed in order to ascertain the variables which can really affect online educational environment in a positive way [4].

Natural feedback on the content being delivered can be taken automatically from learners by using their facial expressions as a tool to measure interestingness of the content and engagement of student in the online lecture [5].Facial expressions can provide critical information on student's interest and participation in online educational learning. Faces provide detailed information about an individual's state of mind, mood and also emotional state. Studies throughout history have shown that facial expressions are the prime representation of human emotions. Facial expressions can be considered as the main source of information, after words, in estimating an individual's thoughts and state of mind [6].

Facial Recognition has proven to be an important tool in automate tutoring as it helps in the improvement of students learning outcomes as well as in the development of the learning experience [7][8]. In the end, this leads to improvement in the learner's involvement in the learning environment.

This research aims at enhancing students' learning outcomes while studying online courses. This can be considered as analyzing the real-time interaction between student and machine, and assessing student's engagement during Elearning session, which is constantly changing over the passage of time. This variable of engagement can be plotted against time and can be considered as a function of time. This engagement function will be called a student's learning curve in the rest of this paper as its variation as a function of time directly affects the learning aptitude and interest of the students.

The basic claim made in this study is that lack of students' involvement/engagement during online classes due to the lack of the physical presence of teachers is the main factor that hinders learners from achieving on-line courses' learning outcomes. This is largely due to the absence of any direct teacher supervision of the students learning process who in such learning context may be distracted in many ways from what they are studying, with there is no one present to supervise them in what they are learning.

A student studying using online resources cannot participate in a verbal communication, then the major attributes that can be observed to ascertain a student's mood and attitude are his facial features and body language [9].

The prime motive behind this study was to devise a methodology to identify major mood patterns with high probability in an e-learning environment. Data continuously pile up when visual data is recorded in real-time. The sample space becomes large and takes more computational power. To address this specific issue, the secondary goal of this research was to integrate a sequential mining technique which can identify mood patterns with high probability. Rules were extracted using Apriori algorithm to reduce the mood sample space by tagging frequent facial feature patterns into predefined five mood categories.

In the subsequent sections, literature is reviewed followed by a discussion of research methodology for applying the proposed technique, and in the end the results are presented with concluding remarks.

\section{LITERATURE REVIEW}

E-learning presents a lot of learning opportunities for people unable to attend regular schools, colleges or universities. Given the importance of E-learning in this information age, a lot of research has been carried out to improve the performance and adaptability of e-learning. This section will present past, present and prospective studies undertaken for the purpose of improving the e-learning ecosystem.

Online teaching and e-learning methodologies have transcended to new levels after the boom of information technology age. As a result, the quality of education and number of online learners has increased substantially. Still, the modernized way of e-learning creates problem that affects a student's learning curve due to unavailability of any direct supervision [10].

An instructor can provide some insight into student's satisfaction during lectures [11], therefore student's involvement in class has direct correlation with the professional aptitude of the instructor[9]. Direct supervision not only facilitates learning but also keeps the student synchronized with the course objectives due to instant communication with the instructor at any time during the lecture. Lack of communication has shown that affected students may experience high levels of frustration [11].

As supervised teaching is very critical to the learning curves of the students, online courses present a different set of challenges to instructors and students. Online students may never visit a physical campus location and may have difficulty establishing relationships with faculty and fellow students. Researchers who study distance learners must understand and account for these differences when investigating student satisfaction [12],mentioned three important types of interaction in online learning courses: (a) learner-content,(b) learnerinstructor, and (c) learner-learner. He emphasized that instructors should facilitate all types of interactions prompting attentiveness in their online courses as much as possible.

E-learning requires use of video, audio, text to simulate the traditional class and learning environment as closely as possible. E-learning environments may be used for a numerous educational purposes. Modern trends indicate that e-learning based education will come at par with traditional education methods in the near future. In an e-learning environment, teacher and student are not in direct interaction and content is provided by the instructor thorough online platforms using multimedia and software interfaces.

As there is no means of instant communication, machine can only understand what it records using standard man machine interfaces. As there is no verbal communication between the student and the e-learning platform, facial expressions are the only means that can provide concrete information about a student's mood and involvement during the class [13]. For example, when students show confused expressions, one of the common mood patterns may be one or a combination of the following facial features i.e. eyebrows 
lowered or drawn together, vertical or horizontal wrinkles on the forehead, and inconsistent eye contact etc. In order to understand whether the student is grasping what is being delivered, a lecturer must sense the subtle nonverbal indicators exhibited by the expressions of the students [14].

Facial features and there relevance to emotions has been rigorously investigated by Ekman et. al [26][27][28][29]in various publications and their work is regarded as one of the most significant contribution to facial attributes based emotion analysis. Facial acting coding system can provide information about instantaneous facial emotional reactions, but still the need to ascertain a complete mood based on various action units as they vary from person to person and situation to situation.

Facial features (Forehead, eyes, nose, mouth, etc.) are the fundamental attributes that are extensively used in face recognition systems as their movements help determine the construction of expression on a human face [15].

Facial recognition can be efficiently used to identify and categorize facial expressions in real-time. Machine learning algorithms have also been employed for facial recognition to enhance accuracy and detection time[16]. Facial expressions are basically emotional impulses translated into physical muscle movements such as, wrinkling the forehead, raising eyebrows or curling of lips. Authors in [17] presented the beneficial prospects of using intelligent methods to extract facial expressions to improve the processing speed of image analysis. Database of facial expressions have been populated in various studies to develop interesting algorithms for various applications.

Emotion recognition study can be broadly categorized into three steps: Face detection, Facial feature extraction and Emotion classification. Detailed research has been carried out in each of these. These three categories are concerned with the central background pertaining to the issue of facial emotion recognition.

In an image, detecting the presence of a human face is a complex task due to the possible differences attributed to different faces. The varying physical attributes of a face are the major cause for this variation. The emotions which are the combination of facial action units [31] in a human face also affect facial appearances.

Neural networks can be actively used to classify a learner's orientation in predetermined categories, which can be associated using Apriori algorithm to allow for real-time HMI intervention for improved involvement. The aim was to assess in real-time whether the e-learning systems can be improvised to recognize the facial expressions and attention state of a learner using classification and data association algorithms. These systems can then be used to improve content delivery of e-learning platforms through real-time mood extraction. Appropriate learning materials and activities for a learner can be incorporated to alter his mood state during e-learning activity.

Face detection can be broadly classified into four categories: knowledge-based approach, feature invariant approach, template-based approach and appearance-based approach [33][34][35][36].

Appearance-based approach maps the human face in terms of a pixel intensities. Since only face patterns are used in its training process, the efficiency is not good. Even the time taken is lengthy, as the number of patterns which needs to be tested is large.

A neural network was found to be quite effective in capturing complex facial patterns from facial images. Both supervised and unsupervised learning approaches are used to train the neural network. Since finding a sufficient training data set is questionable, unsupervised neural networks are more preferable. Apart from neural networks, Support Vector Machines (SVM)[37], eigenfaces, Distribution based approaches, Nave Bayes classifiers, Hidden Markov Models (HMM)[38] and Information theoretical approaches can also be used for face detection in the appearance-based approach [33][34][35][36]. Rather than minimizing the training error as in neural networks, SVM operate by minimizing the upper bound on the generalization error limit instead of minimizing training error as in neural networks. Eigen faces uses Eigen space decomposition and has proven an accurate visual learning method. Nave Bayes classifier is more efficient in estimating the conditional density functions in facial subregions. The HMM differs from template-based and appearance-based approaches as it does not require exact alignment used in these approached rather HMM constitutes a face pattern as a series of observation vectors.

A student involvement in e-learning is directly based on how he can be engaged to focus and listen to the content being delivered. Facial expressions over short instants can be misleading and a time frame based analysis to ascertain emotional states can provide interesting results. For example, confusion and frustration was studied using temporal and order based patterns using continuous affect data [30]. A similar study was carried out by Craig et al [32] which also included boredom. Authors reported that confusion is affiliated with indirect tutor dialogue moves and negative tutor feedback. Similarly, frustration was found to be affiliated with negative tutor feedback, and boredom did not appear to be detectable from the set of three dialogue features [32]. Timing of an emotional state can also play an important role in automated tutoring as reported by authors in [31].This study investigated the relationship between affect and learning. However, identifying the exact places where emotion occurred during the learning process was not covered limiting the efficacy of Auto Tutor system.

\section{RESEARCH OBJECTIVES}

A coherent information exchange between learner and machine is imperative for effective E-learning and is based on the learning curve of the student. Research objectives in this study were formulated to develop a practical technique for understanding student interest during the E-learning session.A student interest can thus be enhanced vide engagement techniques. The research objectives for achieving this objective are listed as follows: 
First objective was to investigate whether facial expressions are the most pertinent means of nonverbal expression mode during e-learning and can in turn assist the e-learning system to identify the interest and comprehension level of the students.

Second objective was to list most common facial features that describe the involvement of a student in a lecture. A list of 54 features were compiled and used in a survey to identify most pertinent facial features for describing student's expression.

The third objective was to develop a methodology to relate facial features to understand expressions of a student during various emotional states describing his involvement in the lecture with high probability in real-time.

Next section consists of the methodology pursued to identify important facial features and will present details on how facial features recorded over certain time frames can provide sufficient information regarding moods of a student in real-time with reduce computational power and sample space.

\section{RESEARCH METHODOLOGY}

Research methodology pursued in this research was conducted phase wise. First, a survey was carried out among instructors involved in e-learning to inquire and identify most probable facial features that represent the facial expressions, and over certain time, the mood patterns of a student. A neural network approach is then used to train the system using facial feature sets to predict specific facial expressions. Data association based algorithm was selected in proposed approach to extract information on emotional states by correlating multiple sets of facial features using support and confidence levels. This was done to improve the clustering of the relevant datasets. The methodology was designed to analyze a student's interest by varying the content being delivered.Different combinations of inter-related facial expressions for specific time frames were used to estimate mood patterns and subsequently level of involvement of a student in an e-learning environment.

A trained dataset of facial features representing student's emotional state is the primary requirement to assess a student's involvement in an e-learning environment. The data has to be collected first, correlated with emotional indicators and is then reused as training data to extract different expressions describing facial features. The data association algorithm is applied to relate features into expression over a time period to discover negative mood patterns of a student during online lecture. The methodology presented here in order to pursue above objectives consists of three major phases, which are as follows:

\section{A. Categorization of Facial Features Using a Survey Instrument:}

Before embarking on a detailed investigation into efficacy of observing facial features to asses a student's interest during an online lecture. A survey is conducted to evaluate whether facial feature analysis is the most pertinent means of understanding a student's behavior during e-learning. Secondly, the survey recorded observations from academics regarding which facial features partially describe mood or emotional state of a student.

In order to construct a baseline for the facial features, a survey was conducted in 2014/2015 academic year and 198 academics from various universities were approached for their response. Experts in online instruction were approached with minimal 2 years of teaching experience at postsecondary level.

Survey was forwarded with a brief explanation of the research objectives with two instrument questions which were,

- Will the process of measuring the learners' degree of engagement/involvement during studying online courses help in learner to focus more and as a result improve the learning outcomes? Which facial expressions you think are most obvious and recurrent in lectures?

- List the most pertinent facial features for eyes, eyebrows, lips and head that constitute facial expressions of student, representing his state of mind and involvement during a lecture.

\section{B. Training and Classification of Facial Features Dataset using Neural Networks:}

Classification algorithms make use of supervised learning techniques to predict the class of previously unobserved data by using a training model from existing data [18]. An efficient way to define a classification model is to characterize it as a set of comprehensive classification rules to provide relevancy and accuracy, simultaneously. An extensive 'Cohn-Kanade' data was selected for training and classification of our neural network model.

The Cohn-Kanade AU-Coded Facial Expression Database [19] is available for research purposes online and is used in facial image analysis and for perceptual studies. This database consists of 486 sequences from 97 faces. Each sequence starts with a neutral expression, gradually leading to the peak

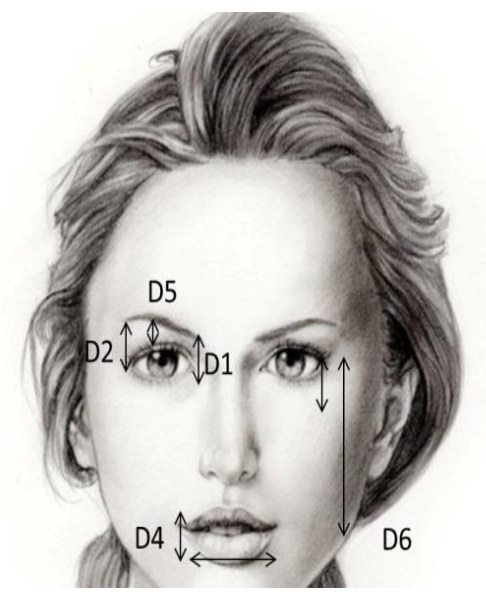

Fig. 1. Distance attributes helping in measuring facial features

expression. This database provides solid foundation for our trained NN model, speeding up the face recognition process.

Feature collection can be carried out using feature extraction algorithms presented in literature. Recognition and 
interpretation mood or learning attitude of a student is carried out by analyzing facial features during the lecture. Neural networks are used to train our system on 'Cohn-Kanade' facial expression database and same was used to identify a student's involvement state during an online lecture. Facial features were characterized into four main categories based on the response of the survey: eyes, eye brows, lips, head (incl.hand/fingers on face).

A Radial basis function NN algorithm [20]which was used in this study to classify facial expressions based on facial features(Table-1). Figure-1 shows the distance points for eyes, eyebrows and lips that were used to define facial features for the training of NN algorithm. Any change in the distance metrics point to a certain facial feature instance and combination of these facial features can be used to classify the five facial expressions.

As distance provides certain thresholds to make decisions related to facial expressions, these thresholds can be used to classify unknown patterns [25]. Figure-2 shows the higher level decision model of NN used in our proposed model for classification of facial features.

In this figure the pixels and combinations of two distance attributes are shown which provide information on eyebrow position relative to the eye. The matrix shown here can be used to plot a feature if D1 and D2 are plotted over a 2-D plot. The proposed classification model is already trained to identify the affinity of the region belonging to the acquired D1 and D2 values, and it correlates the D1 and D2 values to a certain feature, i.e., in this case Feature ' 21 ' based on the prior training data. Therefore it is necessary to train the proposed model with a larger database to improve the probability of detection of a certain feature.

\section{Mood Extraction Using Facial Features}

Associative Data Mining is considered as an important data mining technique and it has been extensively researched and used for data mining by researchers. Data Association helps in mining of association based rules between items based on item set transactions and it is regarded as an important tool for rule discovery in very large datasets [21]. Data association can provide an estimate of unknown relationships and decision rules in a dataset which can greatly improve the process of decision making and prediction [22].

A student's mindset can be well communicated through his facial expressions during an online lecture. Change of mood of a student can be observed using following instruments: facial expressions, hands and body language. These instruments can be observed individually or in combination, however, in both the cases, the data association patterns can be extracted to get a better understanding of the behavior of the student during online learning. This data association approach is very efficient and provides accurate result in instances when one category alone cannot be used to assess accurate understanding of the state of mind of a student [24]. A combination of facial feature categories which have a high probability of occurrence reduces the decision space by many folds. Facial expressions for each student for every online course can amount to very large data. Therefore, a well-established algorithm is presented here to extract moods from a large set of facial features. The algorithm is modified to be used for identifying moods of students and subsequently making accurate decisions about their interest level during the delivery of an online course. Mood extraction using data association is carried out in two stages [24] [25].

\section{If $D 1<5$ AND D2 $>3$ \\ AND \\ If $D 1>2$ AND $D 3<5$ \\ THEN Feature'21' = TRUE}
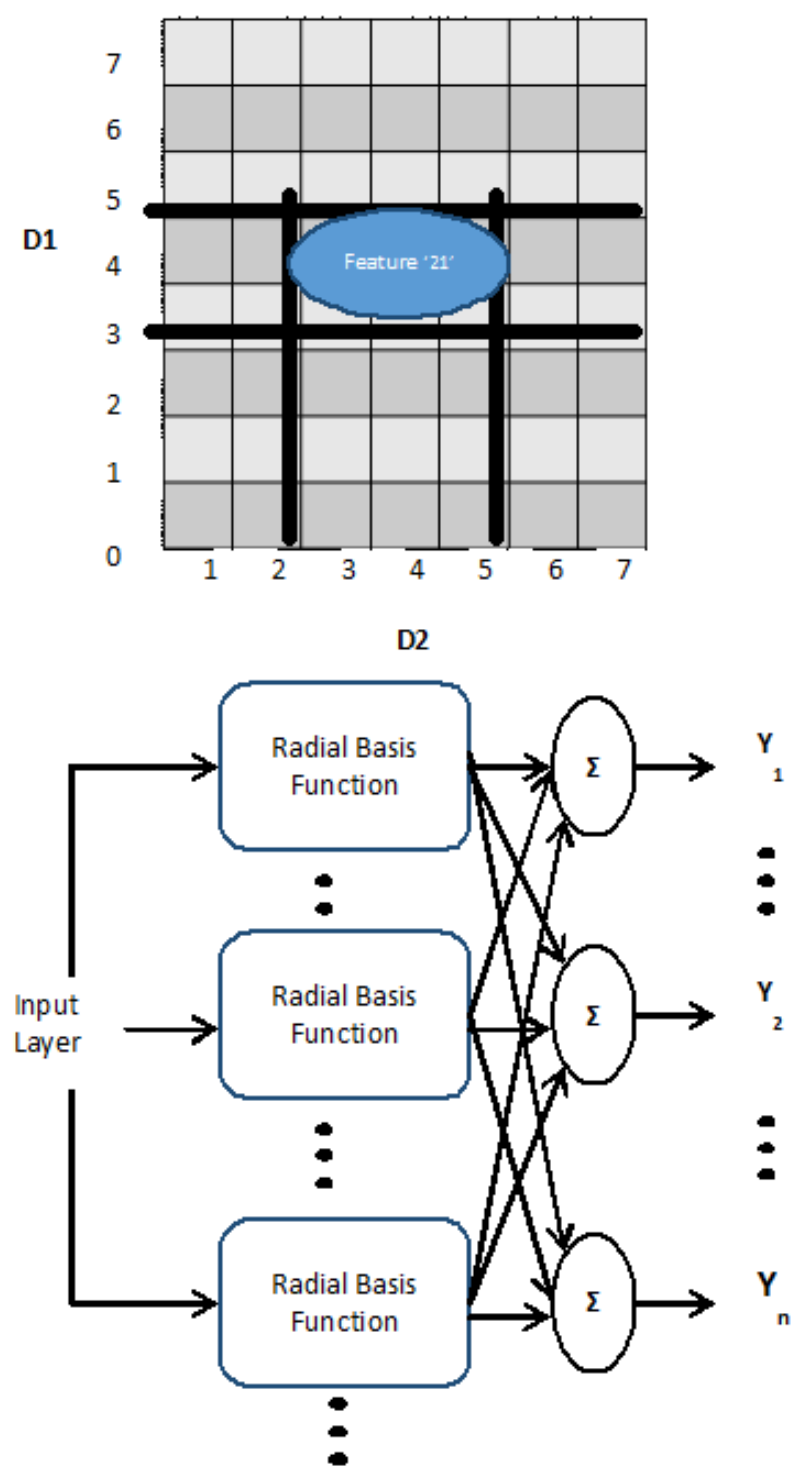

Fig. 2. A distance based radial basis function decision approach for facial features

Approximating categories or item-sets, that occur frequently, and data association for extracting rules that are based on the relationships between these items is the first step of classification. In the subsequent stage,, items are evaluated to segregate sets of items that occur frequently and have a ratio of occurrence greater than the minimum support threshold [23]. 
In the second phase, all possible rules are extracted from the item set, and the number of rules will depend upon the all possible combinations of the items in a given item set e.g. if an item set is of the form $\{a 1, a 2, a 3\}$, then the rules that can be extracted are $\{a 1 \rightarrow a 2, a 3\},\{a 2 \rightarrow a 1, a 3\},\{a 3 \rightarrow a 1, a 2\},\{a 1$, $\mathrm{a} 2 \rightarrow \mathrm{a} 3\},\{\mathrm{a} 1, \mathrm{a} 3 \rightarrow \mathrm{a} 2\},\{\mathrm{a} 3, \mathrm{a} 2 \rightarrow \mathrm{a} 1\}$ etc.

A rule $\{X \rightarrow Y\}$, where $X$ and $Y$ are facial features can be verified using confidence and support threshold levels. Support and confidence thresholds are used as constraints or limits for rule extraction. Support and confidence thresholds provide a measure for pruning the rules which does not meet the threshold criterion. In a nutshell, associative data mining is used for mood extraction by employing user specified support and confidence levels for related facial features and this approach can be used to devise a gauge forassessing extent of correlation between facial features in a dataset.

Apriori algorithm [24] is an efficient technique for data association which can be used to generate frequent feature sets from database facial features. The algorithm [25] makes iterative estimation of most frequent items based on support and confidence metrics. Other metrics may also be used but these are the standard metrics in assessing frequency of an item set and objectiveness of a student's mood during online learning.

Support level is used to estimate that how often a relationship is established in between various facial features in a dataset, while confidence level provides a measure to determine the frequency of ' $\mathrm{B}$ ' facial feature in observed features also containing feature ' $A$ ' during time ' $t$ '. Time period for observing a student's involvement and attentiveness is dependent upon the content being delivered and significant parts from the content that require complete attention from the students for understandability.

Support determines how frequent is a distance attribute appearing in SET ' $A$ ' also appear in SET ' $B$ ' for a given number of samples, whereas, confidence determines how frequently distance attributes from SET ' $B$ ' correlates with distance attributes in SET 'B'.

Abovementioned support and confidence metrics can be mathematically represented as

Support:

$$
\mathrm{s}(\mathrm{A} \rightarrow \mathrm{B})=\sigma(\mathrm{A} \cup \mathrm{B}) / \mathrm{N}
$$

Confidence:

$$
c(A \rightarrow B)=\sigma(A \cup B) / \sigma(A)
$$

Following steps are proposed for robust mood extraction architecture to evaluate interest and attention of a student towards educational content being delivered online. These steps will be form the basis of the proposed algorithm for mood extraction using facial features.

- Frequent Feature-sets Generation: Considering N transactions, all frequent feature-sets are estimated based on support levels. This is an iterative process to identify and generate candidate feature-sets. This part of the algorithm involves two phases. In first phase, it checks each feature-set starting from single facial feature from the feature-set to the maximum size feature-set. In the second phase, new feature-sets are estimated from the previous iteration and support is tested against the support threshold. Number of iterations in this step depends upon the maximum size of item set i.e. $(\operatorname{kmax}+1)$ is the total number of iterations and kmax is the largest size of a frequently occurring feature-set.

- Candidate Generation and Pruning: In this step, new candidate feature-sets are generated based on the (k-1) feature-sets found in the previous iteration followed by pruning by using support levels.

- Support Counting: In this step, occurrence frequency of candidate feature-sets after pruning is determined and support levels are updated.

- Mood Extraction: A level-wise approach is used to discover rules based on data association between consequent and antecedent facial features in frequent feature-sets. At first, all the rules with a single consequent are selected to generate new candidate rules. The selection of these rules is based on respective confidence levels. Rules generated by Apriori algorithm can be large in number depending upon the database being searched.

As a test case, 30 students from a mathematics class were observed during a one hour session of e-learning and expressions were extracted using Apriori method explained above. Lecture session was divided into 10 minutes subsessions, where each sub-session addressed a particular mathematics problem. Table-1lists the 10 minute divisions of a one hour lecture.

TABle I. Modules TAught During 6 X 10 Minute Time Frame in ONE HOUR SESSION

\begin{tabular}{|l|l|}
\hline S.No & Sub-session \\
\hline 1 & Introduction \\
\hline 2 & Matrices \\
\hline 3 & Matrices Multiplication \\
\hline 4 & Matrices Division \\
\hline 5 & $2 \times 2$ Matrix operations \\
\hline 6 & $4 \times 4$ Matrix operations \\
\hline
\end{tabular}

Students with an average age of 15 years were selected for the study. All students were from grade 10 in a private school. Students were selected based on their academic performance and sufficient exposure to e-learning environments. No prerequisite information was provided to them regarding nature of this exercise.

A $35 \mathrm{~mm}$ digital camera was used with a $10 \mathrm{fps}$ frame rate to record facial features. Using association between facial features, facial expressions were sought out to extract mood of a student for $6 \times 10$ minutes time frame during learning. Radial basis function based NN algorithm [20] was employed to classify moods based on facial features. Apriori algorithm is subsequently used to create frequent feature sets or mood sets from which most pertinent rules can be extracted to declare a mood pattern valid. 
A written feedback was acquired from every student after each 10 minutes session comprising of the following two questions:

1) Mention in which parts of the lecture you were

- Happy

- $\mathrm{Sad}$

- Confused

- Disturbed

- Surprised

2) Which parts of the 10 minute frame did you not understand or were inattentive (1 to 10)?

Based on this topology all three phases were executed sequentially, and results for the study are presented in the next section

102 out of 200 participants provided their feedback based on which result of the survey were formulated and was used to categorize five major expressions and 23 facial features. These are listed in Table-2 and Table-3.

An astounding 88 percent of the respondents agreed that facial expressions do reveal the involvement of a student in the class and can be used to assess a student's response to the content being delivered. This provided strong basis for our subsequent analysis, which was carried out to classify facial features using neural networks.

TABLE II. FACIAL EXPRESSIONS DEFINING MOOD OF A STUDENT IN A ClaSSROOM

\begin{tabular}{|c|l|l|}
\hline S.No & Facial Expressions & Freq. \\
\hline 1. & Happy & 91 \\
\hline 2. & Sad & 96 \\
\hline 3. & Confused & 94 \\
\hline 4. & Disturbed & 90 \\
\hline 5. & Surprised & 87 \\
\hline
\end{tabular}

TABLE III. Major Facial Features that Constitute the Five Major FACIAL EXPRESSIONS OF A STUDENT

\begin{tabular}{|r|l|l|}
\hline S.No & Facial Features & Freq. \\
\hline 1. & Eyes focused on the screen & 90 \\
\hline 2. & Eyes enlarge & 75 \\
\hline 3. & Eyes shrink & 76 \\
\hline 4. & Eyes rolling & 81 \\
\hline 5. & Eyes blinking & 92 \\
\hline 6. & Eyeshot in contact with screen & 85 \\
\hline 7. & Wide-open eyes & 80 \\
\hline 8. & Lips tight & 67 \\
\hline 9. & Lips reading softly & \\
\hline 10. & Lips-Smile, laugh & 88 \\
\hline 11. & Lips- pointed & 94 \\
\hline 12. & Raised eyebrows & 73 \\
\hline 13. & Lowered eyebrows & 95 \\
\hline 14. & Parting eyebrows & 73 \\
\hline 15. & Joining eyebrows & 87 \\
\hline 16. & Scratching eyebrows & 69 \\
\hline 17. & Head shaking & 91 \\
\hline 18. & Head dropping & 89 \\
\hline 19. & Head nodding & 81 \\
\hline & & 102 \\
\hline
\end{tabular}

\begin{tabular}{|r|l|l|}
\hline 20. & Hands on face or using them as supports & 84 \\
\hline 21. & Scratching on head & 79 \\
\hline 22. & Scratching on ears & 68 \\
\hline 23. & Scratching on nose & 75 \\
\hline
\end{tabular}

Table-4 shows the comparative performance of the Radial Basis Neural Network model [20], Hidden Markov (HMM)[38]model and Support Vector Machine (SVM)[37] model tested in this study. All these algorithms were implemented in Matlab and integrated with LabView vision module to process and classify image data. All the models were trained using facial feature's distance attributes from CohnKanade database and also from a custom database which was populated using facial expressions of sample space of 30 students. The NN model [20] used in this study outperformed HMM and SVM for the Cohn-Kanade database in this study and proved to be most reliable given sample space is large.

Following facial expressions were targeted for training the proposed model.
a) Happy
b) $\mathrm{Sad}$
c) Confused
d) Disturbed
e) Surprised

TABLE IV. CLASSIFICATION ACCURACY IN CUSTOM AND EXISTING DATABASES

\begin{tabular}{|l|l|l|l|l|l|l|l|}
\hline \multirow{2}{*}{ No } & \multirow{2}{*}{$\begin{array}{l}\text { Facial } \\
\text { Expression }\end{array}$} & \multicolumn{3}{|c|}{$\begin{array}{l}\text { Classification Accuracy } \\
\text { on Existing Database }\end{array}$} & \multicolumn{3}{|c|}{$\begin{array}{l}\text { Classification Accuracy } \\
\text { on Custom Database }\end{array}$} \\
\cline { 3 - 8 } & $N N$ & $S V M$ & $H M M$ & $N N$ & $S V M$ & $H M M$ \\
\hline 1 & Happy & $\begin{array}{l}90.1 \\
\%\end{array}$ & $\begin{array}{l}92.2 \\
\%\end{array}$ & $87.2 \%$ & $81 \%$ & $83 \%$ & $74 \%$ \\
\hline 2 & Sad & $\begin{array}{l}88.1 \\
\%\end{array}$ & $\begin{array}{l}85.0 \\
\%\end{array}$ & $82.6 \%$ & $78 \%$ & $80.8 \%$ & $78.2 \%$ \\
\hline 3 & Confused & $\begin{array}{l}80.6 \\
\%\end{array}$ & $\begin{array}{l}78.2 \\
\%\end{array}$ & $73.4 \%$ & $77 \%$ & $64.5 \%$ & $72.2 \%$ \\
\hline 4 & Disturbed & $\begin{array}{l}86.2 \\
\%\end{array}$ & $\begin{array}{l}81.0 \\
\%\end{array}$ & $80 \%$ & $72 \%$ & $76.3 \%$ & $69 \%$ \\
\hline 5 & Surprised & $\begin{array}{l}85.3 \\
\%\end{array}$ & $\begin{array}{l}84.7 \\
\%\end{array}$ & $84 \%$ & $72 \%$ & $71.4 \%$ & $75 \%$ \\
\hline
\end{tabular}

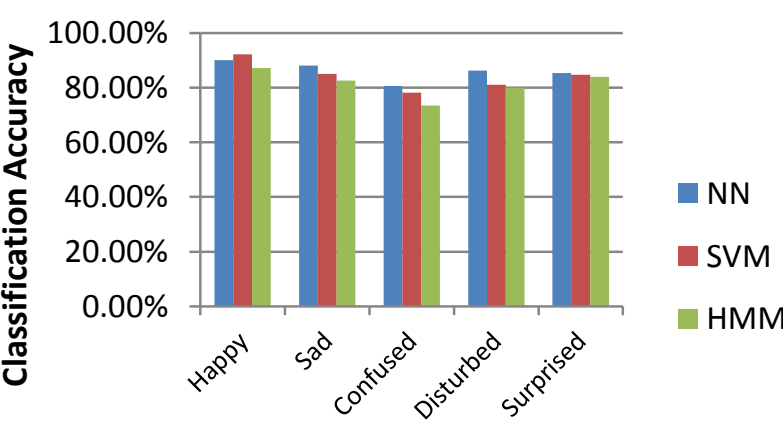

\section{Existing Database}

\section{STUDENT EXPRESSIONS}

Fig. 3. Classification accuracy on existing database 


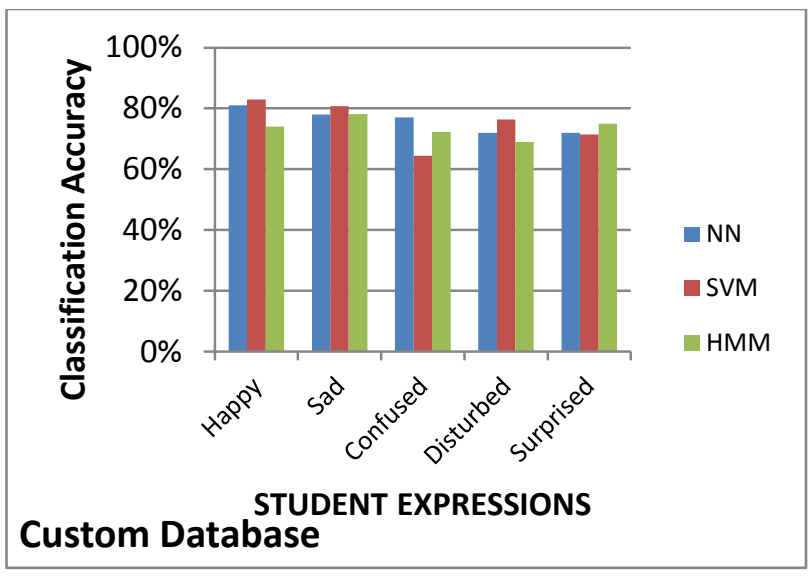

Fig. 4. Classification accuracy on custom database

Based on different distance sets, feature sets were populated and tested for reliability using Cohn-Kanade dataset. Expressions were readily classified with high accuracy, when test images were selected from the same database used for training our NN model. However, accuracy dropped but to acceptable level when custom image set of 30 students was used as a test data set. This problem can be circumvented by using a custom template for images and iteration of neural network training with on new datasets. A similar trend was observed for the SVM and HMM classification carried out on the Cohn-Kanade dataset. For the custom dataset, SVM and HMM results showed random classification rates which can be attributed to small sample space of facial features.

The response of the students was recorded by asking them to provide a score out of 10 for their attentiveness during each 10 min session and based on their feedback; attentiveness was correlated with the extracted facial expression sequences or simply mood patterns. Mood extraction was carried out during every $10 \mathrm{~min}$ session of the one hour mathematics lecture for all the 30 students. The total extracted mood patterns using Apriori and correct patterns based on the correlation results are shown in Table-5

TABLE V. VAlid Mood Patterns Extracted During 6 X 10 Minute TIME FRAME IN ONE HOUR SESSION FOR 30 STUDENTS

\begin{tabular}{|l|l|l|l|}
\hline Mood/Expression & $\begin{array}{l}\text { Extracted } \\
\text { Mood } \\
\text { Patterns }\end{array}$ & $\begin{array}{l}\text { Correct } \\
\text { Mood } \\
\text { Patterns }\end{array}$ & $\begin{array}{l}\text { Percentage } \\
\text { Error }\end{array}$ \\
\hline Happy & 122 & 92 & $75.4 \%$ \\
\hline Sad & 110 & 87 & $79.0 \%$ \\
\hline Confused & 125 & 89 & $71.2 \%$ \\
\hline Disturbed & 109 & 78 & $71.5 \%$ \\
\hline Surprised & 98 & 82 & $83.6 \%$ \\
\hline
\end{tabular}

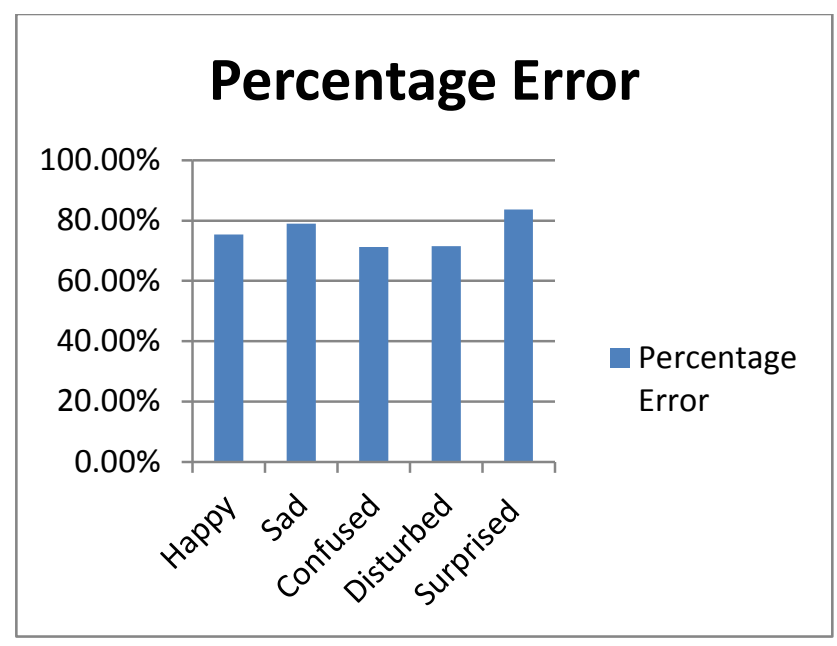

Fig. 5. Percentage error in Mood/ Facial expression classification

Results showed that mood patterns extracted had a high correlation with the feedback provided by the students. In all the cases, our proposed algorithm showed a success rate of over $70 \%$ in assessing the student's mood. This showed that a student's expression over a 10 minute timeframe can be used to predict and extract a student's mood, which in turn can be used to assess student's attentiveness in the class. The results showed that the proposed approach was very robust due to integration of neural network based classification and Apriori algorithm for mood extraction. The difference in success rates for each mood can be related to basic test settings, incomplete database and simpler NN training settings.

The proposed classification and mood extraction method do not attempt to address complete theory of emotions in context of e-learning, rather it is intended to devise a methodology in identifying any mood that is persistent is affecting a student's attention in an e-learning environment, which is an important consideration as highlighted in [31][32] for boredom, confusion and frustration states. The results provided in this research shows that the proposed technique is promising in assessing five moods in an active e-learning environment which were selected using a survey. The success percentage for assessing each emotional state is above $70 \%$. In future work, more emotional states can be tested and based on the results from this study, a similar success ratio is expected given an extensive facial feature database is used.

\section{CONCLUSION}

The art of understanding how different students comprehend educational content during an online study session requires detailed investigation on the behavior and emotional 
state of the student throughout the lecture [33][34]. This research was carried out to determine possible ways to observe and analyze behavior of a student with an aim to understand the events triggering his emotional detachment during an online class.

Visual data acquired using high definition cameras contains a lot of information when stored over a long period of time, and it needs to be continuously recorded thus accumulating into very large data. Data mining approaches can help in similar can help in mining patterns from such large data. Important rules based on correlation characteristics of classification attributes like distance can be acquired to characterize mood swings and changes that affect the learning curves of a student in an e-learning environment. The results can help in better understanding the complete eco-system of an E-learning environment where learner-machine active interaction and raised level of student's engagement is of prime concern. The delivery of e-learning content as well as attitude discrepancies in a student can be then adequately addressed to enhance the student's involvement and attentiveness during eleaning. This can be done during or after the e-learning session based on the preference of the student and/ or the E-learning administrator

Main contribution of this research is the integrated approach with neural network facial recognition and Apriori based mood extraction, which showed a probability of over $70 \%$ for detecting 5 emotional states or moods.

Facial expressions describe the emotional state of the learner and analysis of content and delivery methods can be carried out to achieve optimal experience in an e-learning environment [35]. However it is difficult to devise universal standard content delivery systems for every learner, therefore, specific testing sessions may be incorporated in an e-learning system to allow customization as per student's learning curves.

The results assimilated using a survey response from various academics showed that facial features are the best method to observe changes in the mood of a student and relevant causes can be extracted by relating the timeline with the content delivery and student's successive changes in facial expressions. The problem addressed in this study is limited to determining how a mood can be extracted by associating feature sets comprised of various facial expressions. The cause of alterations in mood and mental state are altogether another problem and is not discussed in this research.

Mental state of a student can be observed using his facial expressions as facial features tend to change and provide the best depiction of what student have in mind [36]. As involvement of a student during unsupervised learning is critical in improving his learning potential, it is pertinent to learn the problems faced by students in an e-learning environment.

Finally, most contribution of this research lies in the results which showed that facial expressions extracted using neural networks, and the reduction of sample space using Apriori algorithm can be actively used to derive student's emotional state during content delivery in an e-learning system. The proposed integrated approach showed a high probability of positive mood detection rate $(>70 \%)$ for five moods. Happy, sad, confused, disturbed, and surprised moods or emotional states

For future work, the resultant data can be used to optimize e-learning content delivery to engage learner more actively in real-time when a mood leading to inattentiveness is detected.

\section{REFERENCES}

[1] G. Eason, B. Noble, and I. N. Sneddon, "On certain integrals of Lipschitz-Hankel type involving products of Bessel functions," Phil. Trans. Roy. Soc. London, vol. A247, pp. 529-551, April 1955. (references)

[2] M. Nur-Awaleh and L. Kyei-Blankson, "Assessing E-learning and student satisfaction in a blended and flexible environment," 2010 International Conference on Information Society, London, 2010, pp. 481-483.

[3] C. Leghris and R. Mrabet, "Cost Comparison of E-Learning Solutions," 2006 7th International Conference on Information Technology Based Higher Education and Training, Sydney, NSW, 2006, pp. 817-824.

[4] Fresen, J. (2007). A taxonomy of factors to promote quality websupported learning. International Journal on E-Learning, 6(3), 351-362.

[5] J. Yu, "An Infrastructure for Real-Time Interactive Distance E-Learning Environment," 2009 First International Conference on Information Science and Engineering, Nanjing, 2009, pp. 3219-3222.

[6] Mohamed Sathik M, Sofia G (2011) Identification of student comprehension using forehead wrinkles. 2011, International Conference on Computer, Communication and Electrical Technology (ICCCET), pp 66-70.

[7] N. Fragopanagos and J. G. Taylor, -2005 Special Issue: Emotion recognition in human-computer interaction. Neural Networks, Neural May 2005. Networks-Special Issue: Emotion and Brain, vol. 18, pp. 389-405.

[8] Rothkrantz, L.J.M. E-learning in virtual environments, Communication \& Cognition, Vol 42, No. 1\&2, pp 37-52, 2009.

[9] A. Walia, N. Singhal and A. K. Sharma, "A Novel E-learning Approach to Add More Cognition to Semantic Web," 2015 IEEE International Conference on Computational Intelligence \& Communication Technology, Ghaziabad, 2015, pp. 13-17.

[10] Fabri, M., Moore, D.J., Hobbs, D.J (2004) "Mediating the Expression of Emotion in Educational Collaborative Virtual Environments: An Experimental Study", in International Journal of Virtual Reality, Springer Verlag, London

[11] M. Feidakis, T. Daradoumis, S. Caballé and J. Conesa, "Measuring the Impact of Emotion Awareness on e-learning Situations," 2013 Seventh International Conference on Complex, Intelligent, and Software Intensive Systems, Taichung, 2013, pp. 391-396.

[12] R. Nkambou, (2006) “Towards Affective Intelligent Tutoring System", Workshop on Motivational and Affective Issues in ITS. 8th International Conference on ITS 2006, pp 5-12

[13] Chaffar, S. and Frasson, C. (2005). "The Emotional Conditions of Learning”. Proceedings of the FLAIRS Conference 2005, .pp. 201-206

[14] Guey-Shya Chen and Min-Feng Lee, "Detecting emotion model in elearning system," 2012 International Conference on Machine Learning and Cybernetics, Xian, 2012, pp. 1686-1691.

[15] Bailenson J, Beall A, Blascovich J, Raimundo M, Weishbush M (2000) "Intelligent agents who wear your face: User's reactions to the virtual self" Technical Report, Center for the Virtual Environment and Behaviors Department of Psychology, University of California, Santa Barbara

[16] R. Brunelli and T. Poggio, "Faical Recognition: Features versus Templates," IEEE Trans. Pattern Analysis and Machine intelligence, vol. 15, no.10, pp. 1042-1052, Oct. 1993.

[17] LA. Essa and A.P. Pentland, "Coding, Analysis, Interpretation, and recognition of facial expressions,"IEEE Trans. Pattern Analysis and Machine Intelligence, vol. 19, no.7, pp-757-763, July 1997

[18] J.W.Grzymala-Busse, "On the unknown attribute values in learning from examples," in Proceedings of the ISMIS-91, 6th International 
Symposium on Methodologies for Intelligent Systems, Lecture Notes in Artificial Intelligentce, Vol.542,Springer-Verlag, Berlin Herdelberg New York,1991,pp.368-377.

[19] G. Zhang, "Neural networks for classification: a survey", IEEE Trans. Syst., Man, Cybern., Syst., vol. 30, pp. 1094-6977, Nov 2000.

[20] Consortium.ri.cmu.edu, 'Cohn-Kanade ( $\mathrm{CK}$ and $\mathrm{CK}+$ ) database Download Site', 2015. [Online]. Available: http://www.consortium.ri.cmu.edu/ckagree/. [Accessed: 20- Sep- 2014]

[21] Weihua Wang, "Face Recognition Based on Radial Basis Function Neural Networks," Future Information Technology and Management Engineering, 2008. FITME '08. International Seminar on , vol., no., pp.41,44, 20-20 Nov. 2008

[22] J. Han, M. Kamber,"Data Mining: Concepts and Techniques", Morgan Kaufmann Publishers, Book, 2000.

[23] F. H. AL-Zawaidah, Y. H. Jbara, and A. L. Marwan, "An Improved Algorithm for Mining Association Rules in Large Databases," Vol. 1, No. 7, 311-316, 2011

[24] T. C. Corporation, "Introduction to Data Mining and Knowledge Discovery", Two Crows Corporation, Book, 1999

[25] R. Srikant, "Fast algorithms for mining association rules and sequential patterns," UNIVERSITY OF WISCONSIN, 1996.

[26] S. Rao, R. Gupta, "Implementing Improved Algorithm Over APRIORI Data Mining Association Rule Algorithm", International Journal of Computer Science And Technology, pp. 489-493, Mar. 2012

[27] Ekman, P. \& Oster, H. (1979). Facial Expressions of Emotion. Annual Review of Psychology, 30, 527-554.

[28] Ekman, P., Friesen, W. V., \& Ancoli, S. (1980). Facial Signs of Emotional Experience. Journal of Personality and Social Psychology, 39(6), 1125-1134.

[29] Ekman, P. (1993). Facial Expression and Emotion. American Psychologist, 48(4), 384-392.
[30] Ekman, P. \& Keltner, D. (1997). Universal facial expressions of emotion: An old controversy and new findings. In Segerstråle, U. C. \& Molnár, P. (Eds.), Nonverbal communication: Where nature meets culture (pp. 27-46). Mahwah, NJ: Lawrence Erlbaum Associates.

[31] Aghababyan, Ani. "E3: Emotions, Engagement and Educational Games." InEducational Data Mining 2014. 2014.

[32] Craig, S. D., Graesser, A., Sullins, J., \& Gholson, B. (2004). Affect and learning: An exploratory look into the role of affect in learning. Journal of Educational Media (now: Learning, Media \& Technology), 29, 241250.

[33] D’Mello, S. K., Craig, S. D., \& Graesser, A. C. (2009). Multimethod assessment of affective experience and expression during deep learning. International Journal of Learning Technology, 4, 165-187.

[34] T. V. Pham, M. Worring, and A. W. M. Smeulders. Face detection by aggregated bayesian network classifiers. Pattern Recogn. Lett., 23(4):451-461, 2002

[35] Li Xia, "Facial Expression Recognition Based on SVM," in Intelligent Computation Technology and Automation (ICICTA), 2014 7th International Conference on , vol., no., pp.256-259, 25-26 Oct. 2014

[36] S. Deshmukh, M. Patwardhan and A. Mahajan, "Survey on real-time facial expression recognition techniques," in IET Biometrics, vol. 5, no. 3, pp. 155-163, 92016.

[37] A. W. P. Fok, H. S. Wong and Y. S. Chen, "Hidden Markov Model Based Characterization of Content Access Patterns in an e-Learning Environment," 2005 IEEE International Conference on Multimedia and Expo, Amsterdam, 2005, pp. 201-204.

[38] W. Gong and W. Wang, "Application research of support vector machine in E-Learning for personality," 2011 IEEE International Conference on Cloud Computing and Intelligence Systems, Beijing, 2011, pp. 638-642. 